\title{
THE EFFECT OF MUTATION ON LINK CONCORDANCE, 3-MANIFOLDS AND THE MILNOR INVARIANTS
}

\author{
JAE CHOON CHA
}

\begin{abstract}
We study the effect of mutation on link concordance and 3-manifolds. We show that the set of links concordant to sublinks of homology boundary links is not closed under positive mutation. We show that mutation does not preserve homology cobordism classes of 3-manifolds. A significant consequence is that there exist 3-manifolds which have the same quantum SU(2)-invariants but are not homology cobordant. These results are obtained by investigating the effect of mutation on the Milnor $\bar{\mu}$-invariants, or equivalently the Massey products.
\end{abstract}

\section{InTRODUCTION AND MAIN RESUlts}

Let $L$ be a link in $S^{3}$. An embedded 2-sphere $S$ in $S^{3}$ is called a Conway sphere for $L$ if $S$ meets $L$ transversally at four points. By cutting $S^{3}$ along $S$ and reglueing along an orientation preserving involution on $S$ which preserves $L \cap S$ setwisely and does not fix any points in $L \cap S$, we obtain a new link in $S^{3}$ with the same number of components as $L$. It is called a mutant of $L$. If $L$ is oriented and the orientation of $L$ is preserved, it is called a positive mutant.

Many invariants fail to distinguish links from their (positive) mutants. Skein invariants including the Alexander, Jones and HOMFLY polynomials, are preserved by mutation, although some colored versions of them are known to distinguish some knots from positive mutants [31]. S-equivalence classes of knots are preserved by positive mutation (for a proof, see [19]). Consequently, all invariants derived from Seifert matrices, including the Alexander module, torsion invariants and signatures, are also preserved.

The problem of distinguishing knots and links from (posive) mutants up to concordance is even harder. Two links $L_{0}$ and $L_{1}$ in $S^{3}$ are said to be concordant if there is a proper submanifold $C$ in $S^{3} \times[0,1]$ which meets $S^{3} \times\{i\}$ in $L_{i}$ for $i=0,1$ and is an $h$ cobordism between $L_{0}$ and $L_{1}$. A link concordant to a trivial link is called a slice link. For knots, primary concordance invariants are extracted from the infinite cyclic structure of complements, but they do not detect positive mutation. Using the secondary invariants of Casson and Gordon [2, 3], Kirk and Livingston proved that positive mutation alters knot concordance classes [20, 19]. Recently Cochran, Orr and Teichner found new invariants that detect non-slice knots which are indistinguishable from slice knots by any previously known invariants [12]. At the present time, few is known about the effect of mutation on the Cochran-Orr-Teichner invariants.

The concordance theory of links is much more complicated than that of knots. The crucial difficulty is that no "good" structure on link complements is known. Because of

Date: June 10, 2001 (First Edition: December 20, 2000).

2000 Mathematics Subject Classification. 57M25, 57M27.

Key words and phrases. Mutation, Link concordance, 3-manifolds, Milnor link invariants. 
this, links that admit specific extra structures was extensively studied. A link with $m$ components is called a boundary link if there is a homomorphism of the link group into the free group $F$ of rank $m$ that sends an element representing the $i$-th meridian to the $i$-th generator. Generalizing the notion of boundary links, we call a link a homology boundary link if it admits a surjection of the link group onto $F$. Equivalence classes of boundary links and homology boundary links under appropriate concordance that preserves the extra structures were classified by Cappell and Shaneson [1], Ko [21], Mio [30], Duval [13] and Cochran and Orr [11]. A more general one is the $F$-structure defined in [23, 24], which sublinks of homology boundary links admit.

A fundamental question arising in the study of the link concordance theory is what kinds of links are concordant to boundary links, homology boundary links or sublinks of homology boundary links, and in particular, whether the sets of concordance classes of boundary links, homology boundary links and sublinks of homology boundary links are proper subsets of the next ones. In this regard, the relationship of geometric operations on links and the extra structures is of special importance. The (strong) fusion operation was studied as a tool that changes the extra structures [7, 9, 14. Especially, in the early 90's, Cochran and Orr proved that homology boundary links which are not concordant to boundary links can be produced by the strong fusion operation on boundary links [10]. It is known that a similar effect can be achieved by mutation; the author and Ko proved that positive mutation transforms some slice links into sublinks of homology boundary links which are not concordant to boundary links [5, 4]. One of our main results is that the effect of mutation on link concordance classes is much more drastic.

Theorem 1.1. There exist slice links which are transformed by positive mutation into links not concordant to sublinks of homology boundary links.

As a consequence, the set of links concordant to sublinks of homology boundary links is not closed under positive mutation.

Mutation on knots and links is closely related with the following analogue for 3manifolds. By cutting a 3-manifold $M$ along an embedded surface $V$ of genus two and reglueing along an orientation preserving involution on $V$ with orbit space $S^{2}$, we obtain a new manifold, which is called a mutant of $M$. It is well known that the complement and the surgery manifold of a mutant of a knot $K$ are obtained by performing mutation on those of $K$ (at most twice). For the analogue for links, we introduce additional terminologies. Mutation on a link $L$ along a Conway sphere $S$ is called self-mutation if $S$ meets exactly one component of $L$. Otherwise, it is called bi-mutation. Then the above statement about the complement and the surgery manifold is true for self-mutation of a link, and bi-mutation of a link preserving each component (called type $R$ in Section 2).

It is also hard to detect the effect of mutation on 3-manifolds. Many invariants of 3 -manifolds are preserved by mutation. They include the hyperbolic volume [36], the Reidemister torsion [34], the $\eta$-invariant (and the Chern-Simons invariant) [27], the Floer homology [37, 17], the Casson-Walker invariant [18] and several quantum invariants [15, 16, 25, 35. Examples of mutation altering the diffeomorphism type of 3-manifolds are found by Kirk [18], Lickorish [25], Kania-Bartoszynska [15] and Kawauchi [16].

We propose a new technique to detect the effect of mutation on 3-manifolds, using the lower central series. For a group $G$, the lower central series $G_{q}$ is defined inductively by $G_{1}=G, G_{q+1}=\left[G, G_{q}\right]$. We call the quotient groups $G / G_{q}$ lower central quotients. 
Theorem 1.2. The lower central quotients of the fundamental groups of 3-manifolds are not preserved by mutation.

It is well known that the lower central quotients are invariant under homology cobordism. From this we obtain significant consequences of Theorem 11.2; homology cobordism classes of 3-manifolds are not preserved by mutation, and hence there are 3-manifolds which are not homology cobordant but have the same quantum $\mathrm{SU}(2)$ invariants.

The above results on link concordance and 3-manifolds are proved by investigating the effect of mutation on the Milnor $\bar{\mu}$-invariants, or equivalently the Massey products. Throughout this paper, we use Milnor's notation for the invariants. For an oriented ordered link $L$ with $m$ components and a sequence $I=i_{1} \cdots i_{q}$ which consists of integers between 1 and $m$ (denoting components of $L$ ), Milnor defined an integer $\mu_{L}(I)$ which depends on a choice of meridian elements in $\pi_{1}\left(S^{3}-L\right)$, and proved that the residue class $\bar{\mu}_{L}(I)$ of $\mu_{L}(I)$ modulo an integer $\Delta_{L}(I)$ is an isotopy invariant of $L$ [29]. (See Section 2 for details.) We denote $q$ by $|I|$ and call it the weight of $\bar{\mu}_{L}(I)$. Stallings proved that $\bar{\mu}_{L}(I)$ is an invariant under $I$-equivalence, and hence, under link concordance 39. Turaev [41], Porter [33] and Stein [40] proved that the $\bar{\mu}$-invariants are equivalent to the Massey cohomology products associated to specific defining systems in link complements.

In Section 2, the effect of bi-mutation on $\bar{\mu}$-invariants is fully understood as follows. First, we show that bi-mutation along a Conway sphere $S$ preserves $\bar{\mu}(I)$ (up to indeterminancy) if any component disjoint to $S$ is involved in $I$.

Theorem 1.3. Suppose that $L^{*}$ is obtained from a link $L$ by bi-mutation along a Conway sphere which meets the $i, j$-th components of $L$, and $I=i_{1} \cdots i_{q}$ is a sequence such that $i_{k} \notin\{i, j\}$ for some $k$. Then $\mu_{L}(I) \equiv \mu_{L^{*}}(I) \bmod \operatorname{gcd}\left(\Delta_{L}(I), \Delta_{L^{*}}(I)\right)$ for some orientation and order of $L^{*}$.

We remark that the orientation and the order for the mutant are left ambiguous in Theorem 1.3 because there is no natural way to choose them in general. This is not a real problem, since it causes to the $\bar{\mu}$-invariants the mere ambiguity of sign and numbering of components. In particular, it is irrelevant to the vanishing of the $\bar{\mu}$-invariants. Indeed in the proof of Theorem 1.3 we will clearly indicate how to choose an orientation and an order satisfying the conclusion.

If $I$ does not satisfy the hypothesis of Theorem 1.3, we may forget components disjoint to the Conway sphere and may assume that $L$ is a 2-component link without any loss of generality. In this case we show that higher weight $\bar{\mu}$-invariants are not preserved by bi-mutation.

Theorem 1.4. 1. All $\bar{\mu}$-invariants of weight $<6$ for 2 -component links are preserved (up to sign) by bi-mutation.

2. For any even $q \geq 6$, there exists a 2-component slice link $L$ (whose $\bar{\mu}$-invariants vanish automatically) with a positive bi-mutant that has vanishing $\bar{\mu}$-invariants of weight $<q$ but nonvanishing $\bar{\mu}$-invariants of weight $q$.

As a consequence of the proof of Theorem 1.4, it is also shown that positive mutation does not preserves the Cochran invariants [6], which are known to be integer-valued liftings of the specific $\bar{\mu}$-invariants of the form $\bar{\mu}(112 \cdots 2)$.

In Section 3, we show similar results for self-mutation. 
Theorem 1.5. 1. All $\bar{\mu}$-invariants of weight $<6$ for 2-component links are preserved (up to sign) by self-mutation.

2. There is a ribbon link $L$ with a positive self-mutant which has nontrivial $\bar{\mu}$-invariants.

Theorem 1.1 follows the second conclusions of Theorems 1.4 and 1.5, since sublinks of homology boundary links have vanishing $\bar{\mu}$-invariants.

In Section 4, we prove Theorem 1.2 by investigating surgery manifolds of links. We construct mutative pairs of 3-manifolds with the different ninth lower central quotients.

Acknowledgements. The author would like to thank Ki Hyoung Ko and Won Taek Song for helpful conversations.

\section{Bi-MUTATION AND THE $\bar{\mu}$-INVARIANTS}

We begin with the definition of the $\bar{\mu}$-invariants due to Milnor [28, 29]. Let $L$ be an oriented ordered link with $m$-components. We denote the link group $\pi_{1}\left(S^{3}-L\right)$ by $G_{L}$. Milnor proved that for any homomorphism of the free group $F$ on $m$ generators $x_{1}, \ldots, x_{m}$ into $G_{L}$ which sends $x_{i}$ to an element representing the $i$-th meridian, the induced map $F / F_{q} \rightarrow G_{L} /\left(G_{L}\right)_{q}$ is surjective for all $q$, and furthermore, its kernel is the normal subgroup generated by $\left[x_{1}, w_{1}\right], \ldots,\left[x_{m}, w_{m}\right]$, where $w_{i}$ is an arbitrary word sent to an element representing the $i$-th longitude in $G_{L} /\left(G_{L}\right)_{q}$. Consider the Magnus expansion of $F$ into the ring of formal integral power series in noncommutative $m$ variables $X_{1}, \ldots, X_{m}$, which is defined by $x_{i} \rightarrow 1+X_{i}$ and $x_{i}^{-1} \rightarrow 1-X_{i}+X_{i}^{2}-\cdots$. The Magnus expansion of $w_{j}$ is of the form $1+\sum \mu_{L}\left(i_{1} \cdots i_{k} j\right) X_{i_{1}} \cdots X_{i_{k}}$, where the sum runs over all $i_{1} \cdots i_{k}$. Let $\Delta_{L}\left(i_{1} \cdots i_{k} j\right)$ be the greatest common divisor of $\mu_{L}(J)$ where $J$ runs over all sequences obtained by permuting proper subsequences of $i_{1} \cdots i_{k} j$ cyclically. Milnor proved that if $q>k+1$, the residue class $\bar{\mu}_{L}\left(i_{1} \cdots i_{k} j\right)$ of $\mu_{L}\left(i_{1} \cdots i_{k} j\right)$ modulo $\Delta_{L}\left(i_{1} \cdots i_{k} j\right)$ is an isotopy invariant of $L$. We remark that $\bar{\mu}(I)$ is defined for any sequence $I$ since an arbitrarily large $q$ can be chosen.

We will prove Theorem 1.3 using this algebraic definition of $\bar{\mu}$-invariants. Suppose $S$ is a Conway sphere of $L$ which meets two components of $L$. Reordering the components, we may assume that $S$ meets the first two components of $L$. Suppose that $I=i_{1} \cdots i_{q}$, where $i_{k} \neq 1,2$ for some $k$. By the cyclic symmetry of $\bar{\mu}$-invariants [29] and by reordering, we may assume that $i_{q}=3 . S$ bounds two 3 -balls in $S^{3}$. Let $B_{1}$ be the one that contains the third component of $L$, and $B_{2}$ be the other. By reordering again, we may assume that $B_{2}$ contains the last $(m-n)$ components and $B_{1}$ contains the remaining components except the first two components.

Let $F$ be the free group of rank $m$ as before, and let $E$ be the subgroup of $F$ generated by the first $n$ generators $x_{1}, \ldots, x_{n}$. We assert that any homomorphism of $E$ into $H=$ $\pi_{1}\left(B_{1}-L\right)$ that sends $x_{i}$ to an element representing the $i$-th meridian induces a surjection of $E / E_{q}$ onto $H / H_{q}$. It can be proved by an argument similar to Milnor's proof of the analogous result for link groups [29], however, we give a simpler proof using Stallings' theorem [39] as follows. For $i=3, \ldots, n$, choose an arc $\gamma_{i}$ in $B_{1}$ joining a point on the $i$-th component of $L$ and a point on $S$. We may assume that $\gamma_{i}$ 's are pairwisely disjoint and the interior of each $\gamma_{i}$ is disjoint to $L$. Let $X=B_{1}-\left(L \cup \gamma_{3} \cup \cdots \cup \gamma_{m}\right)$. Then $H$ is a quotient group of $\pi_{1}(X)$, and hence the given meridian homomorphism of $E$ into $H$ is lifted to a homomorphism of $E$ into $\pi_{1}(X)$. Since $H_{1}(X)$ is a free abelian group 
generated by meridians and $H_{2}(X)=0$, an isomorphism of $E / E_{q}$ onto $\pi_{1}(X) / \pi_{1}(X)_{q}$ is induced by Stallings' Theorem. The assertion follows.

By the assertion, there is a word $w$ in the first $n$ generators $x_{1}, \ldots, x_{n}$ (and their inverses) which represents the third longitude in $H / H_{q}$. Since the composition $E \rightarrow H \rightarrow$ $G_{L}$ is extended to a homomorphism of $F$ into $G_{L}$ sending generators to meridians, $w$ can also be viewed as a word representing the third longitude in $G_{L} /\left(G_{L}\right)_{q}$. On the other hand, the same argument works for a mutant $L^{*}$ of $L$ which is obtained by a mutation along $S$, provided an orientation and an order of $L^{*}$ are chosen so that the restrictions of them on $L^{*} \cap B_{1}$ coincide with those of $L \cap B_{1}$. Thus the same word $w$ represents the third longitude of $L^{*}$ in $G_{L^{*}} /\left(G_{L^{*}}\right)_{q}$. This completes the proof of Theorem 1.3 .

The rest of this section is devoted to the study of the effect of bi-mutation on the $\bar{\mu}$ invariants of two component links. Suppose that $L$ is an oriented ordered two component link and $S$ is a Conway sphere which intersects both components of $L$ as shown in Figure 1 . An involution on $S$ that produces a mutant of $L$ is isotopic to either one of the followings: the $\pi$-rotations along the axes $F$ and $R$ shown in Figure 1, or the composition of them. The associated bi-mutations will be called bi-mutations of type $F, R$ and $F R$, and the associated bi-mutants will be denoted by $L^{F}, L^{R}$ and $L^{F R}$, respectively. They can be characterized as follows: bi-mutation of type $F$ is the positive mutation, which preserves the orientation and reverses the order of the components of $L$. Bi-mutation of type $R$ reverses the orientation and preserves the order. Bi-mutation of type $F R$ reverses both of the orientation and the order.

Cutting $S^{3}$ along $S$, we obtain two string links denoted by $T_{1}$ and $T_{2}$ in Figure 1. $L$ can be viewed as a connected sum of their closures $\alpha$ and $\beta$. For convenience, we always choose an orientation and an order of a bi-mutant which coincide with those of $T_{1}$. Note that this is irrelevant to the vanishing of $\bar{\mu}$-invariants.

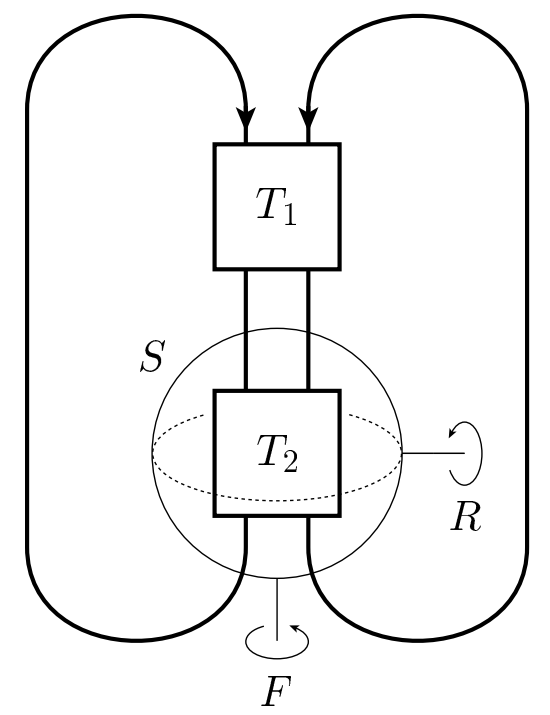

FiguRE 1.

We will describe the $\bar{\mu}$-invariants of the bi-mutants in terms of those of $\alpha$ and $\beta$. For this, we introduce some operations on indices. For a finite sequence $I$ consisting of 1 and 2 , let $I^{F}$ (resp. $I^{R}$ ) be the sequence obtained from $I$ by exchanging 1 and 2 (resp. 
by reversing indices), and let $I^{F R}=\left(I^{F}\right)^{R}$. For example, for $I=112222$, we have $I^{F}=221111, I^{R}=222211$ and $I^{F R}=111122$.

Lemma 2.1. $\quad$ 1. $D(I)=\operatorname{gcd}\left(\Delta_{\alpha}(I), \Delta_{\beta}(I)\right)$ divides $\Delta_{L}(I)$ and

$$
\mu_{L}(I) \equiv \mu_{\alpha}(I)+\mu_{\beta}(I) \bmod D(I) \text {. }
$$

2. For $\tau=F, R$ and $F R, D^{\tau}(I)=\operatorname{gcd}\left(\Delta_{\alpha}(I), \Delta_{\beta}\left(I^{\tau}\right)\right)$ divides $\Delta_{L^{\tau}}(I)$ and

$$
\mu_{L^{\tau}}(I) \equiv \mu_{\alpha}(I)+\mu_{\beta}\left(I^{\tau}\right) \quad \bmod D^{\tau}(I) \text {. }
$$

Proof. Since $L$ is a connected sum of $\alpha$ and $\beta$, the first conclusion follows the additivity of the $\bar{\mu}$-invariants under connected sum (e.g. see [22]).

Fix $q>|I|$, and let $w_{i}\left(x_{1}, x_{2}\right)$ be a word representing the $i$-th longitude of $\beta$ in $G_{\beta} /\left(G_{\beta}\right)_{q}$. For $L^{F}$, the link $\beta^{F}$ obtained by reversing the order of $\beta$ plays the role of $\beta$. $w_{3-i}\left(x_{2}, x_{1}\right)$ represents the $i$-th longitude of $\beta^{F}$. Thus $\bar{\mu}_{\beta^{F}}(I)=\bar{\mu}_{\beta}\left(I^{F}\right)$, and the conclusion for $L^{F}$ follows the additivity again.

For $L^{R}$, the link $\beta^{R}$ obtained by reversing the orientation of $\beta$ plays the role of $\beta$. In this case, the word $v_{i}\left(x_{1}, x_{2}\right)$ obtained by "reversing" the word $w_{i}\left(x_{1}, x_{2}\right)$ (that is, reading from the end to the beginning) represents the $i$-th longitude of $\beta^{R}$. Thus for $I=i_{1} \cdots i_{k} j, \bar{\mu}_{\beta^{R}}(I)=\bar{\mu}_{\beta}\left(i_{k} \cdots i_{1} j\right)=\bar{\mu}_{\beta}\left(I^{R}\right)$ by the cyclic symmetry. This proves the conclusion for $L^{R}$.

The conclusion for $L^{F R}$ follows the above results, since the mutation of type $F R$ is the composition of the mutations of type $F$ and $R$.

Now we are ready to prove the invariance of lower weight $\bar{\mu}$-invariants under bimutation. For 2-component links, it is well known that $\bar{\mu}(12)$ and $\bar{\mu}(1122)$ are all of the nontrivial $\bar{\mu}$-invariants of weight $<6 . \bar{\mu}(12)$ is the linking number, which is preserved by mutation (up to sign because of the ambiguity of an orientation). The indeterminancy $\Delta(1122)$ is equal to the linking number, and hence preserved. We may assume that $\alpha$ has the same linking number as $L$ and $\beta$ has linking number zero, by putting additional full twists on $\alpha$ and $\beta$ which are cancelled in $L$ and $L^{\tau}$. Then we have $\Delta_{\alpha}(1122)=\operatorname{lk}(L)$ and $\Delta_{\beta}(1122)=0$ so that $D(1122)=\Delta_{L}(1122)=\operatorname{lk}(L)=\Delta_{L^{\tau}}(1122)=D^{\tau}(1122)$. By the cyclic symmetry of $\bar{\mu}$-invariants, $\bar{\mu}_{\beta}(1122)=\bar{\mu}_{\beta}\left(1122^{\tau}\right)$ for any $\tau$. Thus $\bar{\mu}_{L}(1122)=$ $\bar{\mu}_{L^{\tau}}(1122)$ by Lemma 2.1. This proves the first conclusion of Theorem 1.4.

Next, we will show that higher weight $\bar{\mu}$-invariants are not preserved by bi-mutation. Explicitly, for any given type $\tau$ we will construct a link $L$ with the following properties, by choosing $T_{1}$ and $T_{2}$ carefully. (1) $L$ is a ribbon link (in particular, all $\bar{\mu}$-invariants of $L$ vanish). (2) For some $I$, all $\bar{\mu}$-invariants of $L^{\tau}$ of weight $<|I|$ vanish but $\bar{\mu}_{L^{\tau}}(I)$ does not vanish.

Take the mirror image of $T_{1}$ (with respect to a horizontal mirror) as $T_{2}$. Then $\beta$ is the mirror image $\alpha^{-1}$ of $\alpha$. Since $L$ is a connected sum of $\alpha$ and its mirror image $\beta, L$ is a ribbon link. For any $\tau=F, R$ and $F R$,

$$
\begin{aligned}
\bar{\mu}_{L^{\tau}}(I) & \equiv \bar{\mu}_{\alpha}(I)+\bar{\mu}_{\beta}\left(I^{\tau}\right) \\
& \equiv \mu_{\alpha}(I)-\bar{\mu}_{\alpha}\left(I^{\tau}\right) \quad \bmod D(I)
\end{aligned}
$$

by Lemma 2.1 and by the fact $\bar{\mu}_{\alpha^{-1}}(I)=-\bar{\mu}_{\alpha}(I)$. Thus if $\alpha$ has vanishing $\bar{\mu}$-invariants of weight $<q$ for some $q$, so does $L^{\tau}$. Furthermore, if $\bar{\mu}_{\alpha}(I) \neq \bar{\mu}_{\alpha}\left(I^{\tau}\right)$ for some $I$ with $|I|=q$, then $\bar{\mu}_{L^{\tau}}(I)$ does not vanish. Thus we are naturally led to the question 
whether the invariants $\bar{\mu}(I)$ and $\bar{\mu}\left(I^{\tau}\right)$ are the same (for links with vanishing $\bar{\mu}$-invariants of weight $<q$ ) for all $I$ with $|I|=q$.

In general, the answer is no so that our construction is successful. A concrete example for type $F$ is obtained by taking as $\alpha$ the link $L_{q}$ suggested by Milnor [29, p. 301] for any even $q \geq 6$. We illustrate $L_{q}$ in Figure 2, where the first and second components are marked as $x$ and $y$, respectively. We take as $T_{1}$ any 2 -string link whose closure is $\alpha$. $L_{q}$ has vanishing $\bar{\mu}$-invariants of weight $<q$, and for $I=1122 \cdots 2(|I|=q)$, $\bar{\mu}_{L_{q}}(I)=(-1)^{q / 2}$ and $\bar{\mu}_{L_{q}}\left(I^{F}\right)=0$ [29, 8]. Thus the links $L$ and $L^{F}$ have the desired properties. In particular, the second conclusion of Theorem 1.4 follows. Furthermore, $L$ and $L^{F R}$ also have the desired properties, since $\bar{\mu}\left(I^{F R}\right)=\bar{\mu}\left(I^{F}\right)$ for $I=1122 \cdots 2$ by the cyclic symmetry.

For type $R$, we need to consider a more complicated example. Take the link given in $₫$, Figure 2.13a] as $\alpha$. Cochran proved that it has vanishing $\bar{\mu}$-invariants of weight $<10$ and nonvanishing $\bar{\mu}(I)$ for $I=2222121211$ in [8, Example 2.12]. The same computational technique proves that it has vanishing $\bar{\mu}\left(I^{R}\right)$. Thus a link with the desired property for type $R$ can be produced by our construction. The author does not know whether there is a simpler $\bar{\mu}$-invariant which is not preserved by bi-mutation of type $R$.

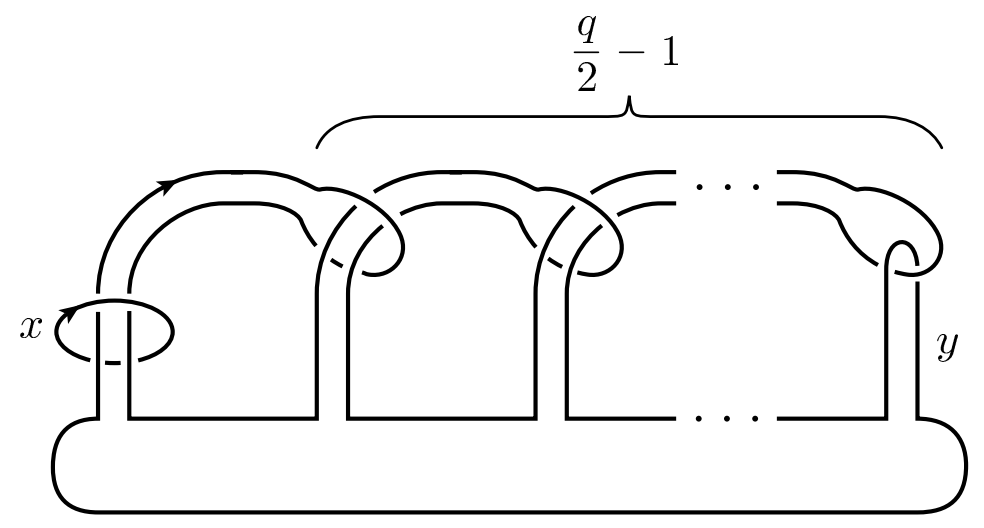

FiguRE 2.

We finish this section with some remarks on the problem of deciding whether $\bar{\mu}(I)$ is invariant under bi-mutation of type $\tau$, or equivalently whether $\bar{\mu}(I) \neq \bar{\mu}\left(I^{\tau}\right)$, for a given $I$. It is closely related to the (not well understood) structure of the space $V_{q}$ of $\bar{\mu}$-invariants of weight $q=|I|$. Milnor found some relations on the $\bar{\mu}$-invariants [29], however, it is not known whether they generate all relations. By Orr, $\operatorname{dim}\left(V_{q}\right)$ was explicitly computed [32]. For $q<6, \operatorname{dim}\left(V_{q}\right)=0$ or 1 and actually all $\bar{\mu}$-invariants of weight $<6$ are preserved by bi-mutation, as it was shown in the above. For most large values of $q$, however, the space $V_{q}$ seems large enough to find an $I$ of weight $q$ such that $\bar{\mu}(I)$ and $\bar{\mu}\left(I^{\tau}\right)$ are independent. On the other hand, Cochran found an algorithm which decides whether $\bar{\mu}(I)$ is nontrivial for a given $I$, (if the answer is yes) computes the least positive value $m(I)$ of $\bar{\mu}(I)$, and constructs a link realizing $m(I)\left[\mathbb{8}\right.$. Since $m(I) \neq m\left(I^{\tau}\right)$ implies $\bar{\mu}(I) \neq \bar{\mu}\left(I^{\tau}\right)$, Cochran's algorithm can be used to obtain partial information on the decision problem. 


\section{Self-mutation and the $\bar{\mu}$-InVARiants}

In this section we investigate the effect of self-mutation on $\bar{\mu}$-invariants for 2-component links. The main tool of this section is the dual interpretation of the Massey cohomology products through the intersection theory of chains. In particular, the following geometric interpretation of Sato and Levine will be used for studying $\bar{\mu}(1122)$. For a 2-component link $L$ with vanishing linking number, each component bounds a Seifert surface disjoint to the other component. We may assume that the two surfaces intersect transversally along a 1-manifold. In [38], it was shown that the self-linking $\beta(L)$ of the 1-manifold along the framing induced by the surfaces is a well-defined link concordance invariant. $\beta(L)$ is called the Sato-Levine invariant. It is well known that $\beta(L)$ coincides with $\bar{\mu}_{L}(1122)$.

Suppose that $L$ is a link with 2-components, and $L^{*}$ is a mutant produced by mutation along a Conway sphere $S$ which meets only one component of $L$. We will show $\bar{\mu}_{L}(1122)=$ $\bar{\mu}_{L^{*}}(1122)$. First we consider a special case of a link $L$ with vanishing linking number. For convenience, we fix an orientation of $L$. (Note that $\bar{\mu}(1122)$ is independent of the choice of an orientation.) Let $x$ be the component of $L$ intersecting $S$, and $y$ be the other component. Let $B_{1}$ be the 3-ball in $S^{3}$ which is bounded by $S$ and disjoint to $y$, and let $B_{2}=S^{3}-\operatorname{int} B_{1}$. Then $x \cap B_{1}$ consists of two oriented arcs $a_{1}$ and $a_{2}$. Choose disjoint oriented arcs $b_{1}, b_{2}$ on $S$ such that $\partial b_{i}=-\partial a_{i}$ and $b_{1} \cup b_{2}$ is setwisely preserved by the involution on $S$ producing $L^{*} . u_{i}=a_{i} \cup b_{i}$ is an oriented simple closed curve. $x \cap B_{2}$ consists of two oriented arcs. Let $c_{1}$ be the one joining the endpoints of $a_{2}$ and $a_{1}$, and $c_{2}$ be the other. See Figure 3, where $T_{1}$ and $T_{2}$ represent a 3 -string link and a 2 -string link, respectively.

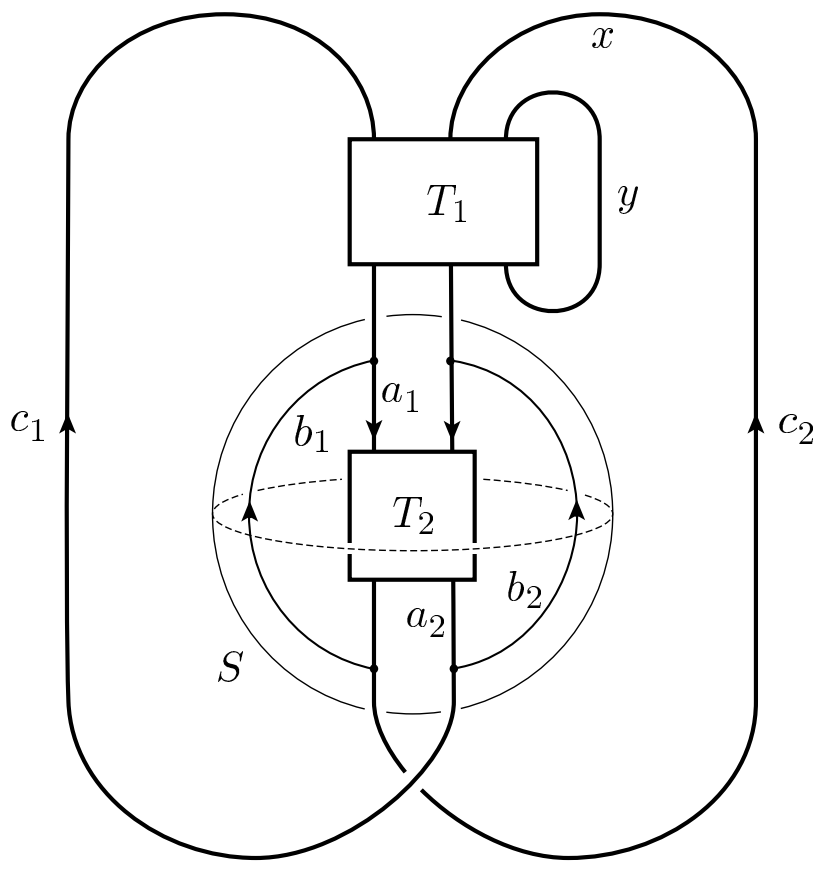

FIGURE 3.

There exist oriented surfaces $E_{1}$ and $E_{2}$ properly embedded in $B_{1}$ and $B_{2}$ which are bounded by $u_{1} \cup u_{2}$ and $\left(-b_{1}\right) \cup c_{1} \cup\left(-b_{2}\right) \cup c_{2}$, respectively. Since the linking number 
of $x$ and $y$ is zero, we may assume $E_{2}$ is disjoint to $y$. Then $V=E_{1} \cup E_{2}$ is a Seifert surface of $x$ which is disjoint to $y$. Choose a Seifert surface $W^{\prime}$ of $y$ in $B_{2}$ which meets $x$ transversally. By deleting an open tubular neighborhood of $W^{\prime} \cap x$ from $W^{\prime}$ and attaching thin cylinders contained in a tubular neighborhood of $x-a_{2}$, a Seifert surface $W$ of $y$ which is disjoint to $x$ is constructed. The Sato-Levine invariant $\beta(L)$ is the self-linking of $c=V \cap W$. We will compute it by evaluating the value of the Seifert form of $V$ at $(c, c)$. $c=\left(E_{1} \cap W\right) \cup\left(E_{2} \cap W\right)$ and $E_{1} \cap W$ is equal to the intersection of $E_{1}$ and attached cylinders, which is a union of parallel copies of $a_{1}$ in $E_{1}$. Therefore $c$ is homologous (on $V$ ) to $k u_{1}+v$ for some integer $k$ and some 1-cycle $v$ in $E_{2}$. See Figure 4 . Thus $\beta(L)=\operatorname{lk}\left(c, c^{\prime}\right)=k^{2} \operatorname{lk}\left(u_{1}^{\prime}, u_{1}\right)+\operatorname{lk}\left(v^{\prime}, v\right)+k \operatorname{lk}\left(u_{1}^{\prime}, v\right)+k \operatorname{lk}\left(u_{1}, v^{\prime}\right)$, where $(\cdot)^{\prime}$ denotes a cycle obtained by pushing slightly along the positive normal direction of $V$. Since $u_{1}$ and $v$ are separated by $S$, the last two terms vanish. Hence $\beta(L)=k^{2} \operatorname{lk}\left(u_{1}^{\prime}, u_{1}\right)+\operatorname{lk}\left(v^{\prime}, v\right)$.

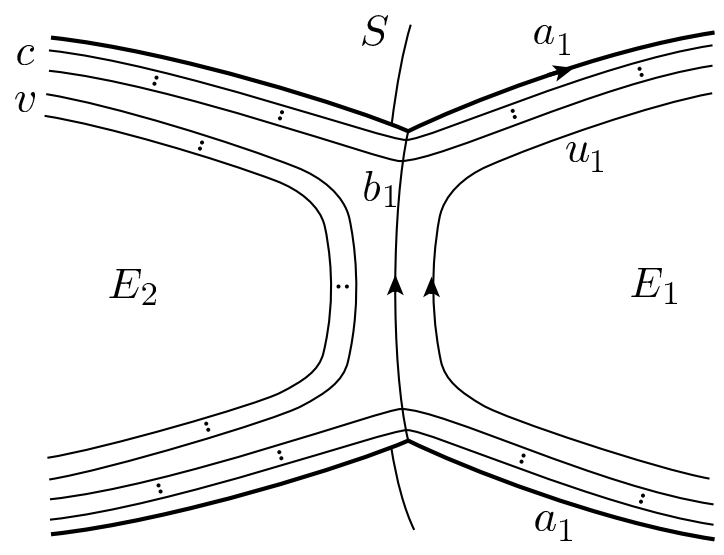

FiguRE 4.

Since $b_{1} \cup b_{2}$ is preserved by the involution on $S$ producing $L^{*}$, a Seifert surface $V^{*}$ of a component of $L^{*}$ in $S^{3}$ is obtained by glueing $\left(B_{1}, E_{1}\right)$ and $\left(B_{2}, E_{2}\right)$ along the involution (we need to reverse the orientation of $E_{1}$ if the mutation is not positive). A Seifert surface $W^{*}$ of the other component of $L^{*}$ such that $W^{*} \cap B_{2}=W \cap B_{2}$ is obtained by attaching cylinders to punctured $W^{\prime}$. As before, the self-linking of $V^{*} \cap W^{*}$ is equal to $k^{2} \operatorname{lk}\left(u_{j}^{\prime}, u_{j}\right)+$ $\operatorname{lk}\left(v^{\prime}, v\right)$, where $j=1$ if the mutation preserves $a_{1}$ setwisely, and $j=2$ otherwise. If $j=1$, we have $\beta(L)=\beta\left(L^{*}\right)$ obviously. If $j=2$, we need additional arguments. Since $u_{1}^{\prime}$ and $E_{1}$ are disjoint, $0=\operatorname{lk}\left(u_{1}^{\prime}, \partial E_{1}\right)=\operatorname{lk}\left(u_{1}^{\prime}, u_{1}\right)+\operatorname{lk}\left(u_{1}^{\prime}, u_{2}\right)$. Similarly $0=\operatorname{lk}\left(u_{2}^{\prime}, u_{1}\right)+\operatorname{lk}\left(u_{2}^{\prime}, u_{2}\right)$. Since $u_{1}$ and $u_{2}$ are disjoint, $\operatorname{lk}\left(u_{1}^{\prime}, u_{1}\right)=-\operatorname{lk}\left(u_{1}^{\prime}, u_{2}\right)=-\operatorname{lk}\left(u_{2}^{\prime}, u_{1}\right)=\operatorname{lk}\left(u_{2}^{\prime}, u_{2}\right)$. This proves $\beta(L)=\beta\left(L^{*}\right)$.

For the general case, choose a link $L^{\prime}$ such that $\operatorname{lk}\left(L^{\prime}\right)=-1 \mathrm{k}(L)$. Choosing basings for connected sums carefully, a connected sum $L \# L^{\prime}$ can be viewed as a self-mutant of a connected sum $L^{*} \# L^{\prime}$. Since $L \# L^{\prime}$ has vanishing linking number, $\mu_{L \# L^{\prime}}(1122)=$ $\mu_{L^{*} \# L^{\prime}}(1122)$. By the additivity of $\bar{\mu}$-invariants, $\mu_{L}(1122)+\mu_{L^{\prime}}(1122) \equiv \mu_{L^{*}}(1122)+$ $\mu_{L^{\prime}}(1122) \bmod \operatorname{lk}(L)$. Thus $\bar{\mu}_{L}(1122)=\bar{\mu}_{L^{*}}(1122)$. This completes the proof of the first part of Theorem 1.5.

The rest of this section is devoted to the proof of the second part of Theorem 1.5. Let $L$ be the link shown in Figure 5. Since $L$ is a connected sum of a link with its mirror image, $L$ is a ribbon link. 


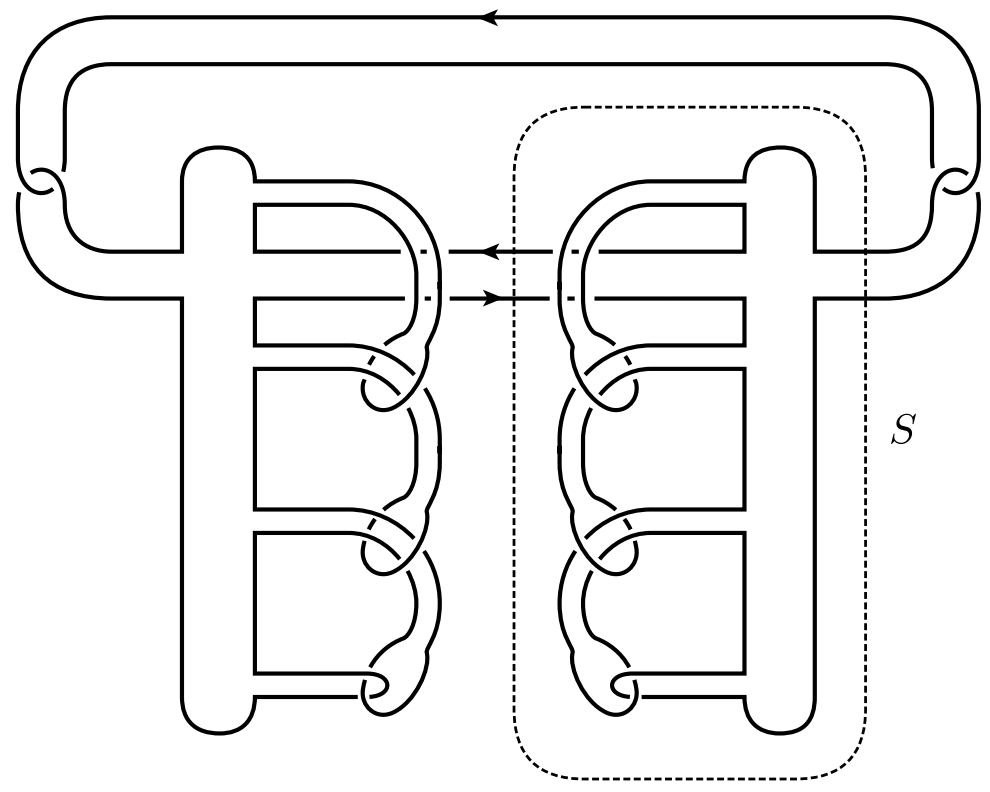

Figure 5.

Let $L^{*}$ be a mutant of $L$ obtained by the positive self-mutation along the Conway sphere $S$ shown in Figure 5. We will compute the $\bar{\mu}$-invariants of $L^{*}$ using a geometric method suggested by Cochran [8]. In the below we describe a reformulated version of Cochran's method where necessary surfaces are constructively obtained.

Suppose that an oriented link with components $x_{1}, x_{2}, \ldots$ such that $\operatorname{lk}\left(x_{i}, x_{j}\right)=0$ for $i \neq j$ is given. Brackets in symbols $x_{1}, x_{2}, \ldots$ are defined inductively as follows. $x_{1}, x_{2}, \ldots$ are called 1-brackets. For a $k$-bracket $u$ and an $(n-k)$-bracket $v, w=(u, v)$ is called an $n$-bracket. $n$ is called the weight of $w$ and denoted by $|w|$. A formal n-linking is defined to be an abstract symbol $\epsilon \operatorname{lk}(w)$, where $w$ is a bracket with weight $n>1$ and $\epsilon=+$ or - . An equivalence relation on formal linkings is generated by two relations $\pm \operatorname{lk}((u, v), w) \sim \pm \operatorname{lk}(u,(v, w))$ and $\pm \operatorname{lk}(w) \sim \mp \operatorname{lk}\left(w^{\prime}\right)$ where $w^{\prime}$ is obtained by replacing a proper (i.e. $w \neq(u, v))$ sub-bracket $(u, v)$ in $w$ by $(v, u)$. If a formal $n$-linking $\pm \operatorname{lk}(u, v)$ has minimal ||$u|-| v||$ in its equivalence class, it is called a minimal n-linking.

We will choose oriented surfaces $V(w)$ and oriented closed 1-manifolds $c(w), c^{\prime}(w)$ associated to brackets, from which the values of formal linkings are defined. For convenience, we will keep the condition that if $w$ and $w^{\prime}$ are as in the second relation in the above, then the curves associated to $w$ and $w^{\prime}$ are the same with opposite orientations, and if $w^{\prime}$ is obtained by replacing a (possibly non-proper) sub-bracket $(u, v)$ in $w$ by $(v, u)$, then the surfaces associated to $w$ and $w^{\prime}$ are the same with opposite orientations. For 1-brackets, let $c\left(x_{i}\right)=x_{i}$ and choose a 0-linking parallel $c^{\prime}\left(x_{i}\right)$ of $x_{i}$ lying on the boundary of a tubular neighborhood $U$ of the given link. Since $\operatorname{lk}\left(c\left(x_{j}\right), c^{\prime}\left(x_{i}\right)\right)=0$, we can choose a Seifert surface $V\left(x_{i}\right)$ of $c^{\prime}\left(x_{i}\right)$ such that $V\left(x_{i}\right) \cap U=c^{\prime}\left(x_{i}\right)$ and $V\left(x_{i}\right)$ and $V\left(x_{j}\right)$ intersect transversally for all $i, j$. Let $c\left(x_{i}, x_{j}\right)$ be the oriented intersection of $V\left(x_{i}\right)$ and $V\left(x_{j}\right)$ (so that the triple of a positive tangent vector of $c\left(x_{i}, x_{j}\right)$ and the positive normal vectors of $V\left(x_{i}\right)$ and $V\left(x_{j}\right)$ induces the orientation of the ambient space) and choose a parallel $c^{\prime}\left(x_{i}, x_{j}\right)$ of $c\left(x_{i}, x_{j}\right)$ along the framing induced by the surfaces. We repeat this process 
as follows. Suppose that $V(u), c(u, v)$ and a parallel $c^{\prime}(u, v)$ of $c(u, v)$ have been chosen for $|u|,|v|<n$ so that the followings are satisfied.

1. All surfaces are in general position.

2. $\partial V(u)=c^{\prime}(u)$ for $|u|<n$.

3. $c(u, v)$ is the oriented intersection of $V(u)$ and $V(v)$ for $|u|,|v|<n$.

4. $c^{\prime}(u, v)$ is a parallel on the boundary of a sufficiently small tubular neighborhood $N(u, v)$ of $c(u, v)$ and both $V(u)$ and $V(v)$ are disjoint to $c^{\prime}(u, v)$ for $|u|,|v|<n$.

5. Unless $V(v)$ contains $c(u)$ or $c^{\prime}(u), N(u)$ is disjoint to $V(v)$ for $|u|,|v|<n$.

In particular, $c(u)$ and $c^{\prime}(u)$ have been chosen for $|u| \leq n$. As a convention, $c(u, u)$ and $c^{\prime}(u, u)$ are defined to be empty. Note that for $u \neq v, V(u) \cap V(v)$ is closed if and only if $c^{\prime}(u) \cap V(v)=V(u) \cap c^{\prime}(v)=\emptyset$. If $\operatorname{lk}\left(c(u), c^{\prime}(v)\right)=0$ for any $|u|,|v| \leq n$, we can choose $V(u)$ bounded by $c^{\prime}(u)$ for $|u|=n$ (and modify $V(u)$ for $|u|<n$ if necessary) so that (5) is satisfied for $|u|,|v| \leq n$. Now $c(u, v)=V(u) \cap V(v)$ is a closed 1-manifold for $|u|,|v| \leq n$, and $c^{\prime}(u, v)$ can be chosen appropriately. This completes the construction of $V(u), c(u, v)$ and $c^{\prime}(u, v)$ for $|u|,|v|<n+1$.

We continue this process until $\operatorname{lk}(c(u), c(v))$ is nonzero for some brackets $u$ and $v$ such that $|u| \leq|v|=n$. At this time we have surfaces $V(w)$ for $|w| \leq n-1$ and curves $c(w)$, $c^{\prime}(w)$ for $w=(u, v),|u|,|v| \leq n-1$. Suppose $q \leq 2 n$. Then in [8, Proposition 2.11] it was shown that $c(u)$ and $c(v)$ exist for any minimal $q$-linking $\operatorname{lk}(u, v)$. We define the value of a formal $q$-linking $\operatorname{lk}(w)$ to be $\pm \operatorname{lk}\left(c(u), c^{\prime}(v)\right)$, where $\pm \operatorname{lk}(u, v)$ is a minimal $q$-linking equivalent to $\mathrm{lk}(w)$. If all linkings of weight $<q$ vanish, the collection of surfaces and curves is called a surface system of weight q. An argument in [8] shows the following.

Theorem 3.1. Suppose that there exists a surface system of weight $q$. Then

1. All $\bar{\mu}$-invariants of weight $<q$ vanish.

2. For $I=i_{1} \cdots i_{q-1} i_{q}$ with $i_{1} \neq i_{q}$,

$$
\bar{\mu}(I)=(-1)^{q} \sum_{w} \operatorname{lk}\left(w, x_{i_{q}}\right)
$$

where the sum runs over all binary parenthesizations on the string $x_{i_{1}} \cdots x_{i_{q-1}}$.

The sum is indeed a linear combination of minimal $q$-linkings, since each summand is equal to a minimal $q$-linking up to sign. Practically, the sum can be computed using the technique of the "formal Massey products" [8]. For example, a straightforward computation shows that if a link with two components $x$ and $y$ admits a surface system of weight 9 ,

$$
\bar{\mu}(122121222)=-20 \operatorname{lk}(y y x y, y x y x y)-20 \operatorname{lk}(y y x y, y y x x y)-20 \operatorname{lk}(y y x y,(y x y, x y))
$$

where $a_{1} \cdots a_{n}$ denotes the bracket $\left(a_{1},\left(\cdots,\left(a_{n-2},\left(a_{n-1}, a_{n}\right)\right) \cdots\right)\right)$.

We apply this method to our example. The link $L^{*}$ admits a surface system of weight 9 . It is best illustrated by figures. Nonempty curves $c(w)$ for $|w| \leq 5$ and surfaces $V(w)$ for $|w| \leq 3$ are illustrated in Figure 6 and Figures 7 [11, respectively. For $|w|=4$, surfaces $V(w)$ can be constructed as in the above discussion since $\operatorname{lk}\left(c(u), c^{\prime}(v)\right)=0$ for $|u|,|v| \leq 4$. We remark that $V(x y)$ in Figure 9 is actually an immersed surface with a self-intersection marked by ----- This is irrelevant in computing the Massey products. Indeed, to avoid the self-intersection, one may take as $V(x y)$ the embedded orientable surface obtained by splicing the immersed surface along the self-intersection. The most complicated part of 
our configuration of surfaces is the relative location of $V(y)$ and $V(x y)$. For the reader's convenience, in Figure 10, we illustrate in detail parts of $V(y)$ and $V(x y)$ in the 3-ball $B$ shown in Figure 9 .

It is straightforward to check that all minimal linkings of weight $\leq 9$ vanish, except $\operatorname{lk}(y y x y,(y x y, x y))=1$. Therefore the $\bar{\mu}$-invariants of weight $\leq 8$ vanish for $L^{*}$, and $\bar{\mu}_{L^{*}}(122121222)=-20$. This proves Theorem 1.5.

We remark that for $n \geq 1$, we can construct a ribbon link and its self-mutant with vanishing minimal linkings of weight $\leq 2 n+7$ except $l \mathrm{k}(y y \cdots y x y,(y \cdots y x y, x y))= \pm 1$, where $\cdots$ represents $(n-2) y$ 's, by modifying our example in Figure 5 in a similar way to the construction of Milnor's link $L_{q}$. The mutant has vanishing $\bar{\mu}$-invariants of weight $<2 n+7$. We conjecture that the nontriviality of the specific $(2 n+7)$-linking implies the nontriviality of some $\bar{\mu}$-invariant of weight $(2 n+7)$. The essential difficulty in the general case is that the technique of the formal Massey products, which was used in our proof for the simplest case, is not suitable for large $n$ since it has exponentially growing computational complexity in $n$. Another interesting question is whether 9 is the minimal weight of the $\bar{\mu}$-invariants not preserved by self-mutation.

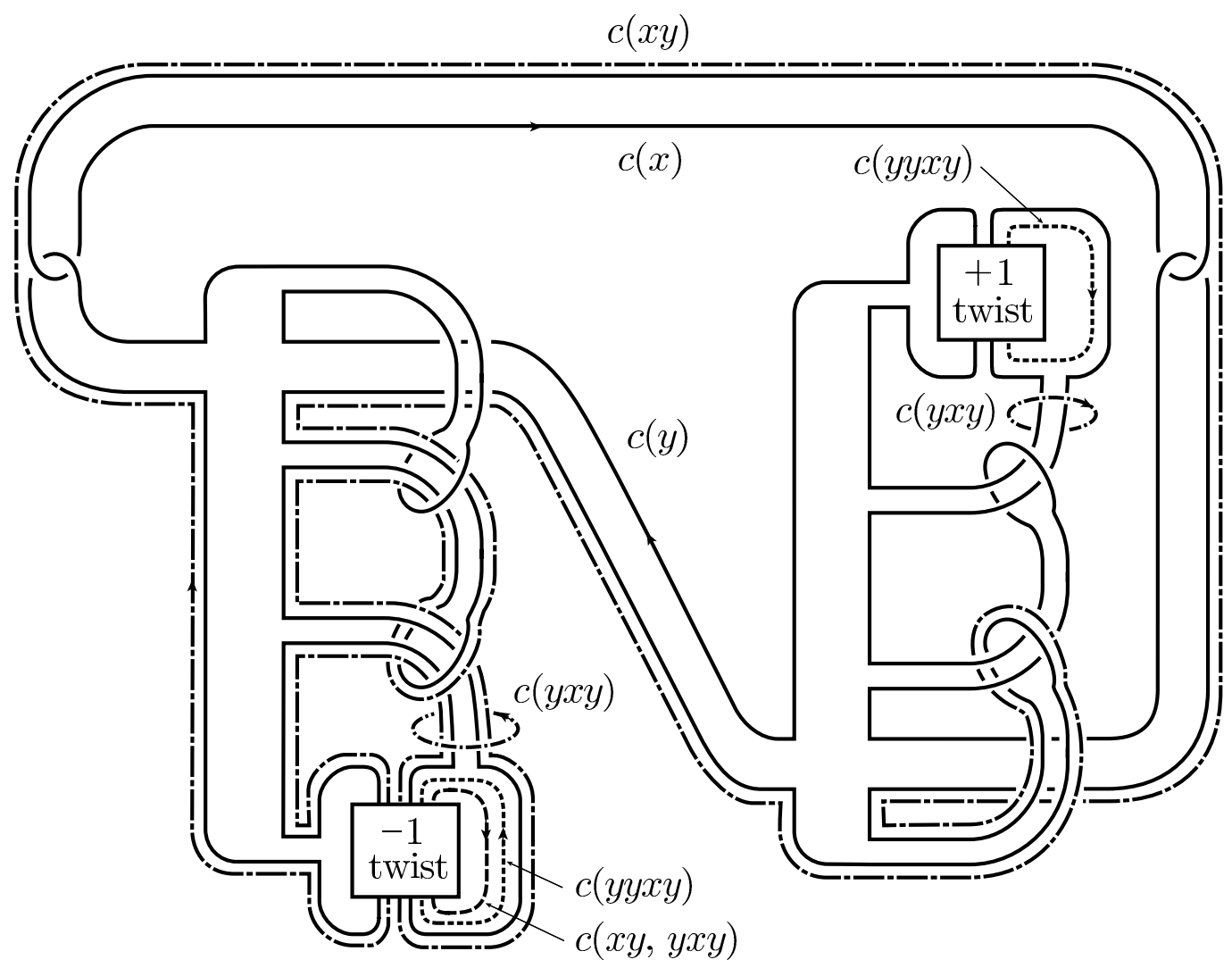

Figure 6. 


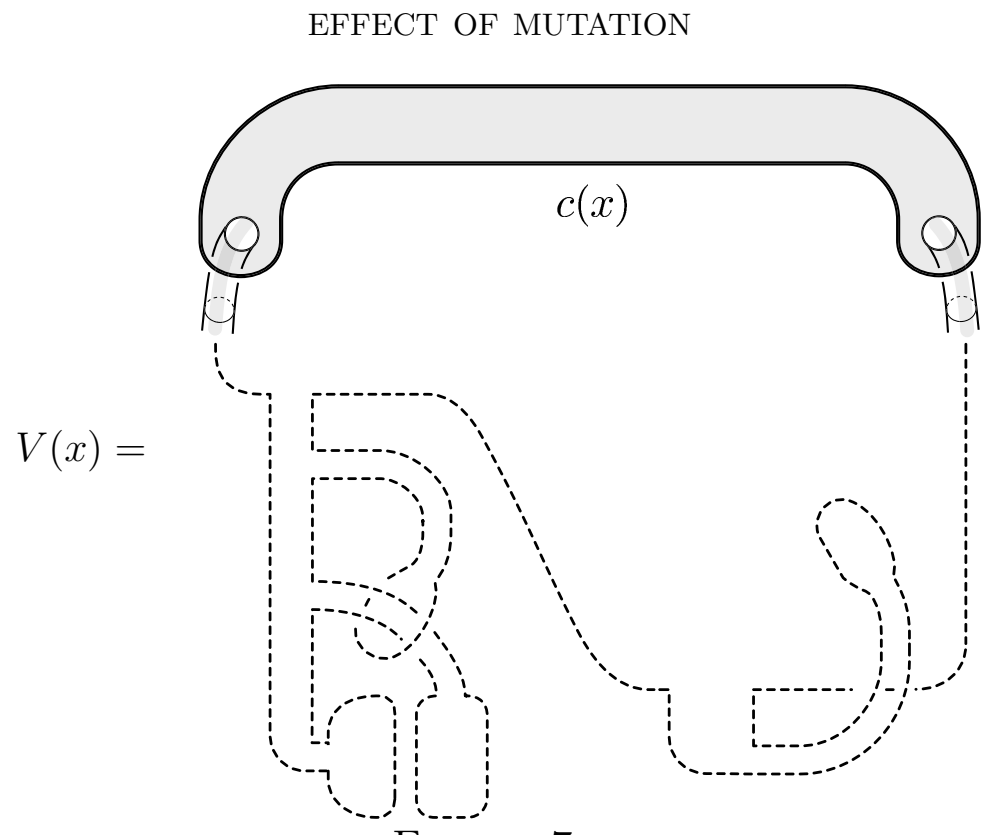

Figure 7.

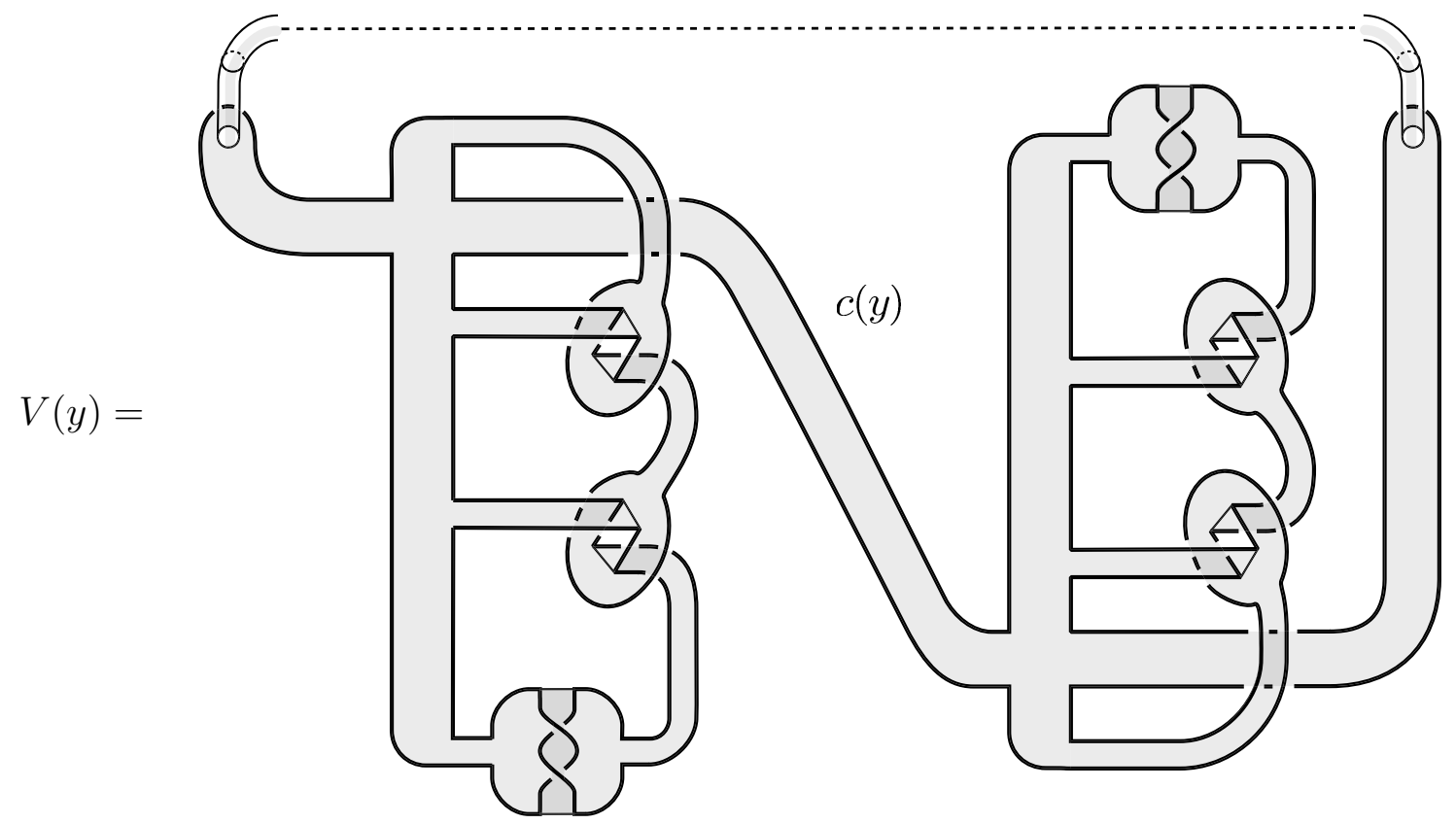

Figure 8. 


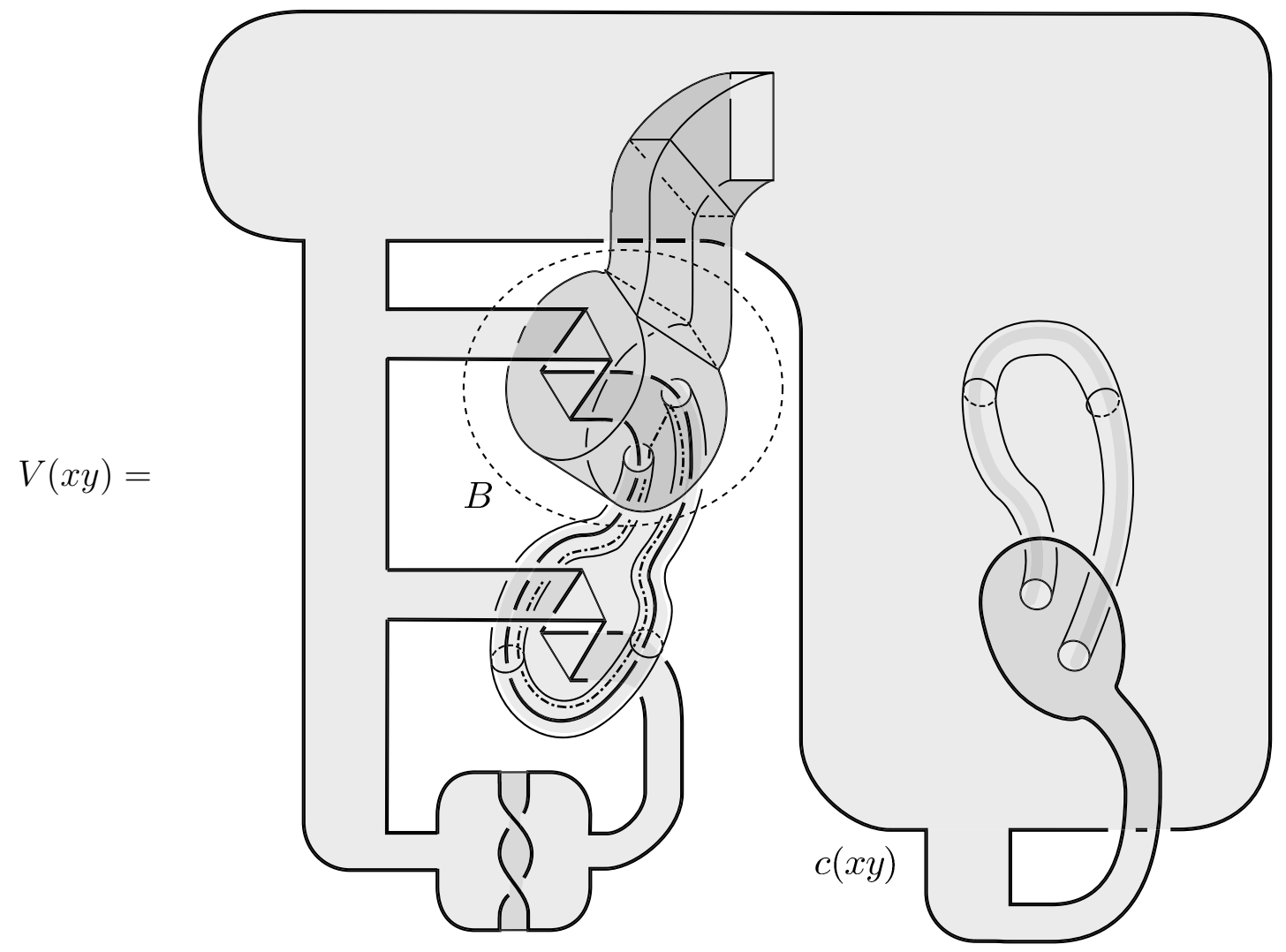

Figure 9.

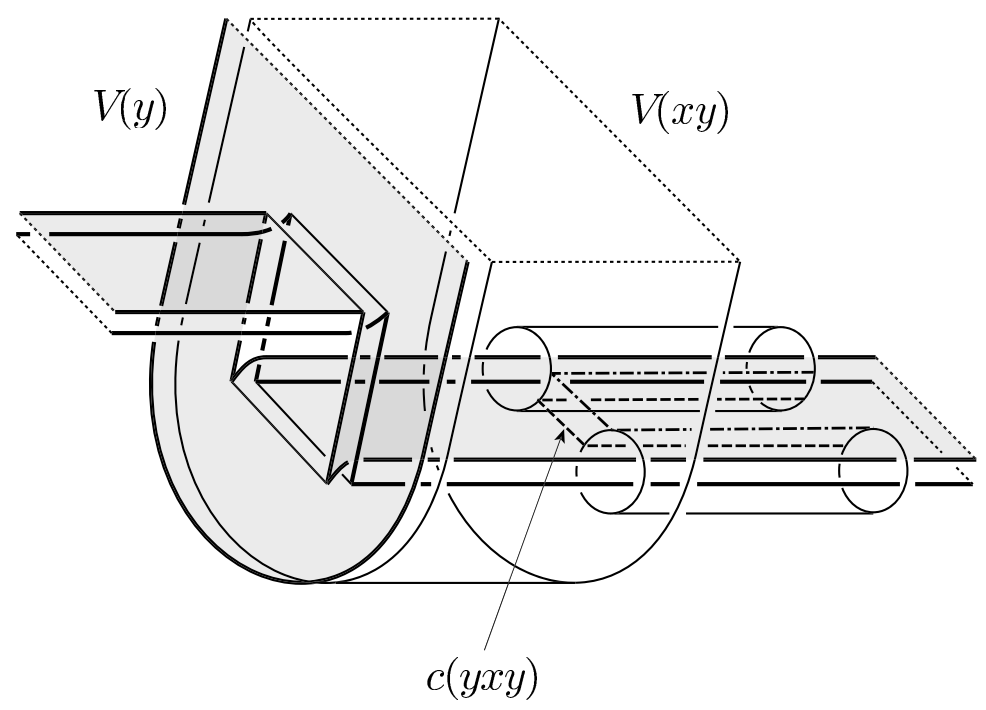

Figure 10. 


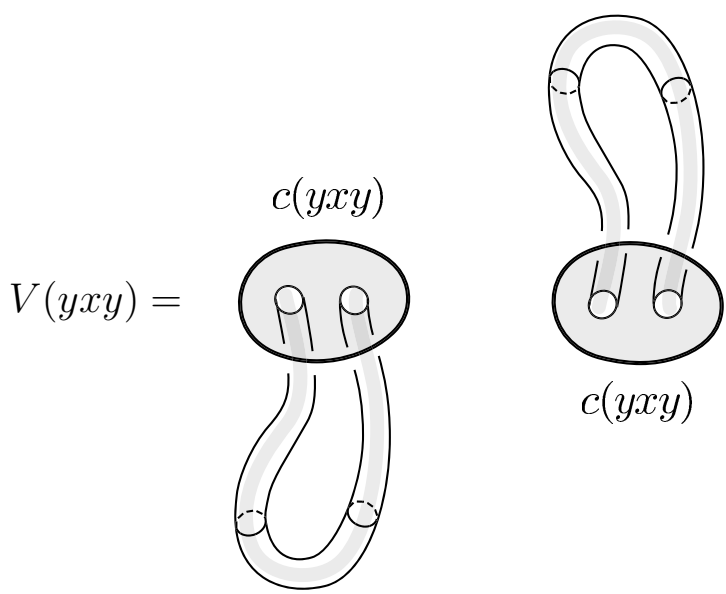

FiguRE 11.

\section{Mutation On 3-MANifolds}

In this section we relate the above results with the effect of mutation on 3-manifolds. Let $L$ be a link with $m$ components in $S^{3}$. By removing an open tubular neighborhood of $L$ and filling with $m$ solid tori so that each 0-linking longitude bounds a disk in a solid torus, we obtain a 3 -manifold $M_{L}$. We call it the surgery manifold of $L$. As expected, the lower central quotients of surgery manifolds are closely related to the $\bar{\mu}$-invariants. Let $F$ be the free group on $x_{1}, \ldots, x_{m}$ as before.

Lemma 4.1. $\pi_{1}\left(M_{L}\right) / \pi_{1}\left(M_{L}\right)_{q}$ is isomorphic to $F / F_{q}$ if and only if all $\bar{\mu}$-invariants of weight $\leq q$ vanish for $L$.

Proof. By Milnor, $G_{L} /\left(G_{L}\right)_{q}$ is isomorphic to $F /\left\langle F_{q},\left[x_{1}, w_{1}\right], \ldots,\left[x_{m}, w_{m}\right]\right\rangle$, where $w_{i}$ is a word in $x_{1}, \ldots, x_{m}$ representing the $i$-th longitude. Since $\pi_{1}\left(M_{L}\right)$ is the quotient group of $G_{L}$ modulo the normal subgroup generated by longitudes, $\pi_{1}\left(M_{L}\right) / \pi_{1}\left(M_{L}\right)_{q}$ is presented as $F /\left\langle F_{q}, w_{1}, \ldots, w_{m}\right\rangle$.

It is well known that all $\bar{\mu}$-invariants of weight $\leq q$ vanish if and only if $w_{1}, \ldots, w_{m}$ are contained in $F_{q}$. If so, the relations $w_{1}, \ldots, w_{m}$ in the above presentation are redundant, and $\pi_{1}\left(M_{L}\right) / \pi_{1}\left(M_{L}\right)_{q}$ is isomorphic to $F / F_{q}$. Conversely, if $w_{i}$ is not contained in $F_{q}$ for some $i$, the normal subgroup $N$ of $F / F_{q}$ generated by cosets $w_{1} F_{q}, \ldots, w_{m} F_{q}$ is nontrivial. Since $F / F_{q}$ is Hopfian (e.g. see [26]), $\pi_{1}\left(M_{L}\right) / \pi_{1}\left(M_{L}\right)_{q}=\left(F / F_{q}\right) / N$ is not isomorphic to $F / F_{q}$.

The following is an immediate consequence of Lemma 4.1.

Theorem 4.1. Suppose that $L$ is a link with vanishing $\bar{\mu}$-invariants of weight $\leq q, L^{*}$ is either a bi-mutant of type $R$ or a self-mutant of $L$, and $L^{*}$ has nonvanishing $\bar{\mu}$ invariants of weight $\leq q$. Then $M_{L^{*}}$ is obtained by performing mutation on $M_{L}$, but $\pi_{1}\left(M_{L^{*}}\right) / \pi_{1}\left(M_{L^{*}}\right)_{q}$ is not isomorphic to $\pi_{1}\left(M_{L}\right) / \pi_{1}\left(M_{L}\right)_{q}$.

In Sections 2 and 3, we found examples of bi-mutation of type $R$ and self-mutation satisfying the hypothesis of Theorem 4.1. Thus Theorem 1.2 follows. 


\section{RefEREnCES}

[1] S. E. Cappell and J. L. Shaneson, Link cobordism, Comment. Math. Helv. 55 (1980), no. 1, 20-49.

[2] A. Casson and C. Gordon, On slice knots in dimension three, Algebraic and geometric topology (Proc. Sympos. Pure Math., Stanford Univ., Stanford, Calif., 1976), Part 2, Amer. Math. Soc., Providence, R.I., 1978, pp. 39-53.

[3] _ Cobordism of classical knots, À la recherche de la topologie perdue, Birkhäuser Boston, Boston, MA, 1986, With an appendix by P. M. Gilmer, pp. 181-199.

[4] J. C. Cha and K. H. Ko, Signatures of covering links, arXiv:math.GT/0108206.

[5] _ Signatures of links in rational homology homology spheres, arXiv:math.GT/0108197, to appear in Topology.

[6] T. D. Cochran, Geometric invariants of link cobordism, Comment. Math. Helv. 60 (1985), no. 2, 291-311.

[7] _ Link concordance invariants and homotopy theory, Invent. Math. 90 (1987), no. 3, 635-645.

[8] _ Derivatives of links: Milnor's concordance invariants and Massey's products, Mem. Amer. Math. Soc. 84 (1990), no. 427, x+73.

[9] T. D. Cochran and J. P. Levine, Homology boundary links and the Andrews-Curtis conjecture, Topology 30 (1991), no. 2, 231-239.

[10] T. D. Cochran and K. E. Orr, Not all links are concordant to boundary links, Ann. of Math. (2) 138 (1993), no. 3, 519-554.

[11] Homology boundary links and Blanchfield forms: concordance classification and new tangletheoretic constructions, Topology 33 (1994), no. 3, 397-427.

[12] T. D. Cochran, K. E. Orr, and P. Teichner, Knot concordance, whitney towers and $L^{2}$-signatures, arXiv:math.GT/9908117, 1999.

[13] J. Duval, Forme de Blanchfield et cobordisme d'entrelacs bords, Comment. Math. Helv. 61 (1986), no. 4, 617-635.

[14] U. Kaiser, Homology boundary links and fusion constructions, Osaka J. Math. 29 (1992), no. 3, 573-593.

[15] J. Kania-Bartoszynska, Examples of different 3-manifolds with the same invariants of Witten and Reshetikhin-Turaev, Topology 32 (1993), no. 1, 47-54.

[16] A. Kawauchi, Topological imitation, mutation and the quantum SU(2) invariants, J. Knot Theory Ramifications 3 (1994), no. 1, 25-39.

[17] _ Mutative hyperbolic homology 3-spheres with the same Floer homology, Geom. Dedicata 61 (1996), no. 2, 205-217.

[18] P. Kirk, Mutations of homology spheres and Casson's invariant, Math. Proc. Cambridge Philos. Soc. 105 (1989), no. 2, 313-318.

[19] P. Kirk and C. Livingston, Concordance and mutation, arXiv:math.GT/9912174.

[20] P. Kirk and C. Livingston, Twisted knot polynomials: inversion, mutation and concordance, Topology 38 (1999), no. 3, 663-671.

[21] K. H. Ko, Seifert matrices and boundary link cobordisms, Trans. Amer. Math. Soc. 299 (1987), no. 2, 657-681.

[22] V. S. Krushkal, Additivity properties of Milnor's $\bar{\mu}$-invariants, J. Knot Theory Ramifications 7 (1998), no. 5, 625-637.

[23] J. P. Levine, Link concordance and algebraic closure. II, Invent. Math. 96 (1989), no. 3, 571-592.

[24] - Link concordance and algebraic closure of groups, Comment. Math. Helv. 64 (1989), no. 2, $236-255$.

[25] W. B. R. Lickorish, Distinct 3-manifolds with all SU(2) invariants the same, Proc. Amer. Math. Soc. 117 (1993), no. 1, 285-292.

[26] W. Magnus, A. Karrass, and D. Solitar, Combinatorial group theory: Presentations of groups in terms of generators and relations, Interscience Publishers [John Wiley \& Sons, Inc.], New YorkLondon-Sydney, 1966.

[27] R. Meyerhoff and D. Ruberman, Mutation and the $\eta$-invariant, J. Differential Geom. 31 (1990), no. 1, 101-130. 
[28] J. W. Milnor, Link groups, Ann. of Math. (2) 59 (1954), 177-195.

[29] - Isotopy of links. Algebraic geometry and topology, A symposium in honor of S. Lefschetz, Princeton University Press, Princeton, N. J., 1957, pp. 280-306.

[30] W. Mio, On boundary-link cobordism, Math. Proc. Cambridge Philos. Soc. 101 (1987), no. 2, 259266.

[31] H. R. Morton and P. Traczyk, The Jones polynomial of satellite links around mutants, Braids (Santa Cruz, CA, 1986), Amer. Math. Soc., Providence, RI, 1988, pp. 587-592.

[32] K. E. Orr, Homotopy invariants of links, Invent. Math. 95 (1989), no. 2, 379-394.

[33] R. Porter, Milnor's $\bar{\mu}$-invariants and Massey products, Trans. Amer. Math. Soc. 257 (1980), no. 1, 39-71.

[34] J. Porti, Torsion de Reidemeister pour les variétés hyperboliques, Mem. Amer. Math. Soc. 128 (1997), no. 612, $\mathrm{x}+139$.

[35] Y. W. Rong, Mutation and Witten invariants, Topology 33 (1994), no. 3, 499-507.

[36] D. Ruberman, Mutation and volumes of knots in $S^{3}$, Invent. Math. 90 (1987), no. 1, 189-215.

[37] - Mutation and gauge theory. I. Yang-Mills invariants, Comment. Math. Helv. 74 (1999), no. $4,615-641$.

[38] N. Sato, Cobordisms of semiboundary links, Topology Appl. 18 (1984), no. 2-3, 225-234.

[39] J. Stallings, Homology and central series of groups, J. Algebra 2 (1965), 170-181.

[40] D. Stein, Massey products in the cohomology of groups with applications to link theory, Trans. Amer. Math. Soc. 318 (1990), no. 1, 301-325.

[41] V. G. Turaev, The Milnor invariants and Massey products, Zap. Naučn. Sem. Leningrad. Otdel. Mat. Inst. Steklov. (LOMI) 66 (1976), 189-203, 209-210, Studies in topology, II.

E-mail address: jccha@knot.kaist.ac.kr

Department of Mathematics, Korea Advanced Institute of Science and Technology, TAEJON, 305-701, KOREA 